\title{
O papel do administrador público nas demandas judiciais no serviço de assistência farmacêutica
}

The role of public administrator in lawsuits related with the pharmaceutical assistance service

\section{Luciane Anita Savi}

Farmacêutica, Doutora em Biotecnologia. Secretaria Municipal de Saúde de Florianópolis. Brasil

\author{
Elisa Silva dos Santos \\ Advogada. Secretaria Municipal de Saúde de Florianópolis. Brasil
}

Resumo: As demandas judiciais na Assistência Farmacêutica, as quais resultam no acesso a medicamentos e insumos por ordem judicial são crescentes, gerando desvio de recursos de outros setores, desorganização e fragilização do serviço, individualização de atendimentos em detrimento do coletivo, e mais ações judiciais, formando um ciclo. Os medicamentos fornecidos por ordem judicial não passam por avaliação de segurança, eficácia e custo/benefício (critérios básicos para padronização pelo SUS), ou mesmo se há real necessidade ou possibilidade de substituição. Parecem razoáveis ações pleiteando medicamentos padronizados quando a falta destes resulta da má gestão ou quando não existe política de tratamento (mesmo havendo terapia eficaz, segura e que o SUS possa financiá-la). Por outro lado, não há razão para ações quando há política pública de tratamento ou que pleiteiam produtos de eficácia e segurança duvidosas, sem indicação de uso e sem registro nos órgãos reguladores. É preciso analisar a origem da judicialização, as ações preventivas, o papel das instituições envolvidas e os desafios do administrador nessas demandas, como nos processos de aquisição dos produtos. É fundamental a inter-relação entre os profissionais do direito e de saúde a fim de equacionar alguns dilemas, como o confronto coletivo $X$ individual. Há que se compreender a judicialização, mensurar sua amplitude e efeitos no SUS e na sociedade. Se por um lado as decisões judiciais visam o direito do cidadão, por outro, remetem ao gestor a responsabilidade de decisões que geralmente contradizem os princípios constitucionais da equidade e acesso à assistência à saúde de qualidade.

Palavras-chave: Assistência farmacêutica; demandas judiciais; acesso a medicamentos.

Key-words: Pharmaceutical assistance; lawsuits; access to medicines 


\section{Assistência farmacêutica e demandas judiciais}

A Assistência Farmacêutica consiste num conjunto de ações voltadas à promoção, proteção e recuperação da saúde, tanto individual como coletiva, tendo o medicamento como insumo essencial. Este conjunto envolve a pesquisa, o desenvolvimento e a produção de medicamentos e insumos, bem como a sua seleção, programação, aquisição, distribuição, dispensação, garantia da qualidade dos produtos e serviços, acompanhamento e avaliação de sua utilização, na perspectiva da obtenção de resultados concretos e da melhoria da qualidade de vida da população (OPAS, 2002; Brasil, 2006). O serviço de Assistência Farmacêutica tem integra ações de promoção, prevenção, recuperação e reabilitação da saúde e apóia as ações de saúde no acesso aos medicamentos essenciais e no seu uso racional (Brasil, 2006).

A implementação de políticas públicas na área de Assistência Farmacêutica contribui para a definição de diretrizes que envolvem os setores público e privado de atenção à saúde, estratégicas para a garantia do direito à saúde estabelecido pela Constituição (Brasil, 1988). Esse direito abrange o acesso ao medicamento adequado, na dosagem e tempo de tratamento corretos e com a orientação necessária a fim de que sua utilização seja resolutiva.

Porém, um fenômeno recente conhecido como "judicialização da Assistência Farmacêutica" ou "judicialização da saúde", o qual consiste na solicitação e obtenção de medicamentos e outros produtos ou serviços da área da saúde por meio de decisão judicial, tem sido freqüente nas Secretarias de Saúde. O grande problema das demandas judiciais é o desvio de recursos de outros setores ou programas para atender a essas ações, levando a uma oferta precária de serviços, que pode resultar em mais demanda judicial, formando assim um ciclo vicioso (Souza et al., 2011). Ao se fornecer medicamentos por ordem judicial, não se avalia se aquele tratamento é o melhor em termos de relação custo/benefício, se o indivíduo realmente necessita do medicamento pleiteado, se pode ser substituído por outro disponível nos programas de Assistência Farmacêutica do Sistema Único de Saúde (SUS) e, ainda, se está sendo infringida alguma lei ou princípio fundamental do sistema de saúde. Apenas se cumpre a ordem determinada pelo juiz (Macedo, Lopes, Barberato-Filho, 2011).

A fundamentação jurídica da grande maioria das ações judiciais para fornecimento de medicamentos está baseada na garantia constitucional do direito à 
saúde. O reconhecimento do direito à saúde tem sido objeto de polêmicas envolvendo políticos, advogados, cientistas sociais, economistas e profissionais de saúde. Discute-se, especialmente, a eficácia do argumento jurídico em relação aos direitos sociais e a avaliação da saúde como bem econômico (Macedo, Lopes, Barberato-Filho, 2011).

O direito à saúde é previsto na Constituição Federal de 1988 em seu art. 6º, integrado no Capítulo II do Título II - Dos Direitos e Garantias Fundamentais, apontado como direito social oponível ao Estado brasileiro, por todos que vivem neste território, e mais especificamente no Título VIII - Da Ordem Social - Seção II, art. 196:

Art. $6^{\circ}$ São direitos sociais a educação, a saúde, o trabalho, a moradia, o lazer, a segurança, a previdência social, a proteção à maternidade e à infância, a assistência aos desamparados, na forma desta Constituição.

Art. 196. A saúde é direito de todos e dever do Estado, garantido mediante políticas sociais e econômicas que visem à redução do risco de doença e de outros agravos e ao acesso universal e igualitário às ações e serviços para sua promoção, proteção e recuperação.

De acordo com a Constituição Federal, o direito à saúde é um direito de toda sociedade, garantido através de Políticas Públicas de Saúde. O SUS representa a principal ferramenta que consolida o direito à saúde com base em três princípios: universalidade do atendimento, equidade e integralidade. Sendo assim, todos os setores de auxílio à saúde devem está balizados nos princípios defendidos pelo SUS, que são garantidos sob a forma de Políticas Públicas (Araújo et al., 2013).

A promulgação da Constituição Federal de 1988 motivou o surgimento das ações judiciais, isso, em função da mesma ter elevado o conceito de saúde a um patamar muito alto, fortalecendo um princípio maior, que é o direito à vida. Dessa maneira, o Poder Judiciário tem examinado pedidos para determinar o Estado a fornecer medicamentos sob a alegação de que a Constituição da República estabeleceu que a saúde é direito fundamental a ser perseguido e implementado, conforme previsão dos arts. 6 e 196 (Araújo et al., 2013).

A Lei Orgânica da Saúde (Lei n 8.080/1990), que disp õe sobre as condições para a promoção, proteção e recuperação da saúde, a organização e o funcionamento dos serviços correspondentes, estabelece que a assistência terapêutica no SUS deve ser integral e ainda incluir a assistência farmacêutica. No SUS, "integral" diz respeito à atuação articulada das ações de promoção, prevenção 
e recuperação da saúde, considerando três níveis de complexidade para essas ações e serviços. Implica em analisar e suprir as necessidades de cuidados de saúde aos indivíduos desde aquelas mais básicas até as mais complexas, garantidas por um conjunto de bens e serviços de saúde de eficácia comprovada no tratamento dos principais problemas epidemiológicos da população brasileira (Vieira, Zucchi, 2009; Medici, 2010).

É possível imaginar as consequências ao SUS se a interpretação do conceito de integralidade for diferente deste apresentado, dado que o mercado de medicamentos e produtos para a saúde é bastante expressivo (cerca de 15.000 medicamentos e 49.000 materiais hospitalares). Se a palavra integral, como qualificação da assistência terapêutica, assumir o significado de "tudo o que há no mercado", então é evidente o que tal interpretação pode trazer para o SUS (Vieira, Zucchi, 2009).

As cortes judiciais no Brasil têm interpretado o tema da integralidade de forma distinta do conceito utilizado pelo Ministério da Saúde. Em grande parte das demandas judiciais, o que acaba ocorrendo é a exigência de se tratar certa doença com o uso de determinado produto farmacêutico, procedimento ou terapia não incorporados pela evidência médica disponível, mesmo que o tratamento desta doença já esteja contemplado no SUS por meio de alternativas terapêuticas comprovadamente mais eficazes pelos parâmetros disponíveis. O caráter difuso do conceito de integralidade tem sido um dos principais fatores que potencializam a iniquidade financeira entre ricos e pobres no acesso ao SUS, acabando por favorecer aqueles que podem pagar por advogados ou que conhecem os meandros jurídicos para levar adiante ações contra o Estado (Medici, 2010).

O interesse da indústria farmacêutica na judicialização da Assistência Farmacêutica não pode ser negligenciado. Das ações judiciais resultam vendas garantidas de medicamentos ao Estado, considerando que, não raro, tais produtos não cumprem os critérios mínimos para serem incluídos nas padronizações oficiais do SUS, e por isso, não são adquiridos para serem fornecidos de acordo com as políticas públicas pertinentes.

A indústria disponibiliza comercialmente milhares de especialidades farmacêuticas. O lançamento constante de "produtos novos" permite à empresa projetar no mercado uma imagem de capacidade tecnológica e científica, 
especialmente junto ao médico. Essa atuação faz parte da estratégia da indústria, na qual as empresas buscam manter sua imagem de inovadoras e garantir sua capacidade competitiva. A maioria desses "novos" medicamentos é, na verdade, fruto de pequenas mudanças nas suas estruturas moleculares que não proporcionam nem representam melhorias ou ganhos substanciais sob o ponto de vista terapêutico (Marin et al., 2003).

Como a inovação gera vultosos lucros, há que se disciplinar, através de processos de validação e normatização pública, sua difusão e comercialização para que esta se baseie em princípios éticos que garantam a eficiência, a eficácia e a efetividade de seu uso em benefício da população. Neste sentido, é necessário estabelecer meios para que os protocolos aceitos como custo-efetivos sejam normatizados e licenciados por instituições acreditadas. A incorporação de novos procedimentos e tecnologias nos sistemas de saúde deve se basear em critérios de necessidade, oportunidade, razoabilidade, conveniência e essencialidade para a garantia da saúde, em seus aspectos coletivos e individuais (Medici, 2010).

\section{Principais fatores envolvidos nas demandas judiciais da assistência farmacêutica}

Quanto às razões que impulsionam a judicialização da assistência farmacêutica, estas variam desde a falta de medicamentos nas farmácias por erros no gerenciamento da assistência farmacêutica até o desconhecimento das listas oficiais de medicamentos oferecidos pelo sistema público por parte dos prescritores e requerentes, passando também pela indicação em desacordo com os critérios estabelecidos nos Protocolos Clínicos e Diretrizes Terapêuticas (PCDT) do Ministério da Saúde (Araújo et al., 2013).

Os profissionais de saúde precisam ter conhecimento sobre a assistência terapêutica disponibilizada e seu acesso pelo SUS, bem como das políticas vigentes para o acesso e uso racional dos medicamentos e insumos. Para tanto, capacitação e formação de recursos humanos na saúde precisam acontecer de forma dinâmica e eficiente (CONASS, 2007; Andrade et al., 2008; Souza et al., 2011; Araújo et al., 2013).

Também, são causas relacionadas ao crescente número das ações judiciais: o modelo médico-assistencial, os hospitais desvinculados da rede, o predomínio das 
ações curativas, o atendimento centrado na medicalização das ações e a pressão para a implantação de novas tecnologias (Araújo et al., 2013).

Outro fator que impulsiona as ações judiciais é a negativa de fornecimento pela falta de documentação ou o não cumprimento de critérios estabelecidos em PCDT. Em algumas situações há predominância de pequena quantidade de advogados e médicos envolvidos em muitas ações, o que pode representar a existência de interesses, além da conquista do direito ao tratamento para o usuário, indicando que o alvo seja a comercialização de novos medicamentos e outros instrumentos terapêuticos que são financeiramente inacessíveis à população em geral (Araújo et al., 2013).

A falta de medicamentos nas unidades de saúde, decorrente de problemas na seleção, programação, aquisição, controle de estoque, armazenamento e distribuição de medicamentos, e a dispensação por trabalhadores sem qualificação legitimam a via judicial como mecanismo de acesso aos direitos previstos na Constituição, especialmente quando estão garantidos por políticas públicas específicas, como a de assistência farmacêutica (Macedo, Lopes, Barberato-Filho, 2011).

A freqüência de solicitações judiciais por medicamentos não padronizados pode estar relacionada à inexistência de alternativas terapêuticas ofertadas pelo SUS, uma vez que as listas são restritivas. A pressão da indústria farmacêutica sobre os prescritores, na tentativa de gerar a necessidade do uso de determinados medicamentos, pode ser outro problema (Chieffi, Barata, 2010; Macedo, Lopes, Barberato-Filho, 2011).

As listas de medicamentos do SUS, muitas vezes, não oferecem opção de escolha no caso de contra-indicação do medicamento prescrito, como, por exemplo, a intolerância a um medicamento em idosos expostos à polimedicação. Esse tipo de demanda pode ser significativamente reduzido com a melhor qualificação dos serviços de assistência farmacêutica, capacitando-os para a avaliação das necessidades individuais de cada paciente e oferecendo mecanismos formais, fundamentados no uso racional, para acolher os pleitos de medicamentos não previstos nas listas do SUS no âmbito da gestão (Macedo, Lopes, Barberato-Filho, 2011).

Em princípio, as demandas judiciais poderiam ser separadas em dois grupos: as justificadas e as injustificadas. Parte das ações judiciais possui por objeto o 
fornecimento de medicamentos que constam nas listas de medicamentos padronizados. Parece razoável e, portanto, justificado que tal demanda se dê por via judicial, caso a falta de acesso aos medicamentos tenha se dado por má gestão da assistência farmacêutica por parte da Secretaria de Saúde, pois o tratamento com o produto farmacêutico demandado está previsto em uma política pública. Assim, como também parece razoável pensar que a ausência de cobertura de determinada doença por uma política de tratamento consista em motivo para considerar essa demanda justificada, desde que exista uma alternativa terapêutica eficaz, segura, disponível nacionalmente e que o sistema de saúde possa financiá-la (Vieira, Zucchi, 2009).

Por outro lado, não parece razoável que os indivíduos demandem medicamentos e produtos para saúde quando há política de tratamento estabelecida e com qualidade, ou que requeiram produtos de eficácia duvidosa, cujo custo é proibitivo ao sistema de saúde, comprometendo, por restrição orçamentária, o acesso de milhares de pessoas a medicamentos. Confundem-se com demandas justificadas a solicitação de: medicamentos sem o devido registro na Agência Nacional de Vigilância Sanitária; medicamentos não incorporados, mas com alternativa terapêutica disponível no SUS, sem contra-indicação para o paciente, e melhor ou tão efetiva quanto aquele pleiteado; medicamentos cuja literatura científica não recomenda a indicação para a qual foram prescritos (Vieira, Zucchi, 2009; Macedo, Lopes, Barberato-Filho, 2011).

\section{Algumas consequências das demandas judiciais da assistência farmacêutica}

O crescimento do número de ações judiciais, associado ao fato de que as decisões são predominantemente favoráveis aos autores, vem gerando problemas para o sistema de saúde como um todo, já que algumas decisões acabam comprometendo gravemente o orçamento para a saúde, em razão do elevado preço de alguns medicamentos pleiteados, que, em muitos casos, não se encontram nos elencos oficiais da regulamentação da assistência farmacêutica no âmbito do SUS (Borges, Ugá, 2010).

No caso das ações judiciais individuais para fornecimento de medicamentos estão em jogo bens providos pelo Estado com recursos públicos e, portanto, a decisão sobre a adjudicação do bem - no caso, o medicamento - afetará, ainda que indiretamente, toda a coletividade. Dessa forma, no caso de soluções que envolvam 
recursos públicos e políticas sociais, torna-se necessária a adoção de critérios racionais e uma análise de cada hipótese posta, não se podendo adotar uma única solução para todas as situações nas quais se requer 0 fornecimento de medicamentos.

Ressalte-se que a decisão de dispensar certo tipo de medicamento pelo Estado envolve critérios técnicos, nos quais se pretende garantir o acesso da população a produtos seguros, eficazes e de qualidade, e ao menor custo possível. Desse modo, a integralidade da assistência à saúde, que inclui a assistência farmacêutica, sob esse ponto de vista, não é um conceito que admite toda e qualquer terapêutica existente ou demandada por um paciente ou profissional de saúde. Há que se ter critérios técnicos, atualizados cientificamente, para embasar a incorporação desta ou daquela tecnologia, desta ou daquela terapêutica ou medicamento. Portanto, da mesma maneira, quando decisões sobre o fornecimento de medicamentos são tomadas na esfera judicial também se faz necessária a utilização de critérios por parte do juiz, que, por não possuir expertise no tema, deve buscar, além dos fundamentos legais aplicáveis ao caso, o uso de critérios que levem em consideração a política de medicamentos planejada pelo Ministério da Saúde e demais órgãos responsáveis em níveis estadual e municipal, encarregados da seleção de medicamentos, a qual é feita por meio de listas oficiais veiculadas em normas específicas ou programas de assistência farmacêutica (Borges, Ugá, 2010).

Segundo Chieffi \& Barata (2009), a análise de processos referentes à dispensação de medicamentos mediante demandas judiciais permitiu evidenciar os efeitos que essas decisões têm sobre a formulação e a execução da política de saúde. Os dados mostram que a parcela da população atendida por demandas judiciais tem em média melhores condições sócio-econômicas, residindo em áreas com baixa ou sem nenhuma vulnerabilidade social. A despeito das intenções de arbitragem de problemas sociais e de defesa dos interesses dos indivíduos diante do poder do Estado, a interferência do Judiciário acaba por atender aos indivíduos que por sua inclusão social já se encontram em posição privilegiada, reforçando ainda mais as desigualdades sociais no campo da saúde. (Albuquerque et al., 2006). 


\section{Aquisição de medicamentos para atendimento das demandas judiciais}

A maior parte das liminares concedidas estabelece um prazo curto para a dispensação do medicamento ao usuário, o qual inclui o tempo previsto para instrução do processo, aquisição, entrega do medicamento ou produto pela empresa e a dispensação ao paciente.

Em se tratando de medicamentos não padronizados e que não integram nenhum Programa e que, portanto, provavelmente não estão disponíveis no estoque da Secretaria de Saúde, as formas mais utilizadas para atendimento no prazo são a aquisição por dispensa de licitação por emergência ou declaração de inexigibilidade por exclusividade, sendo o fundamento legal, respectivamente, o Inciso IV do Artigo 24 e o Inciso I do Artigo 25, ambos da Lei 8666/93, que institui normas para licitações e contratos da Administração Pública (Brasil, 1993; CONASS, 2007).

O artigo 24 da Lei 8666/93 fixa as situações em que é facultado à Administração não licitar:

Artigo 24. É dispensável a licitação: “... nos casos de emergência ou de calamidade pública, quando caracterizada urgência de atendimento de situação que possa ocasionar prejuízo ou comprometer a segurança de pessoas, obras, serviços, equipamentos e outros bens, públicos ou particulares, e somente para os bens necessários ao atendimento da situação emergencial ou calamitosa e para as parcelas de obras e serviços que possam ser concluídas no prazo máximo de cento e oitenta dias consecutivos e ininterruptos, contados da ocorrência da emergência ou calamidade, vedada a prorrogação dos respectivos contratos".

Portanto, existe justificativa legal para que a primeira aquisição para atendimento da determinação judicial seja feita fundamentada nesse dispositivo. Para as compras subsequentes isso não se aplica, devendo a administração instaurar procedimento licitatório sempre que houver possibilidade de competição.

Tanto para os casos de Ação Civil Pública, quanto para ações individuais que determinem o fornecimento de um mesmo medicamento, é recomendável que as aquisições sejam feitas pelo Sistema de Registro de Preços. Isso porque, nesse sistema, a administração estima um quantitativo anual e, ao final da licitação, é assinada uma Ata de Registro de Preços e não um contrato de aquisição. Na medida em que há necessidade de adquirir o medicamento, utiliza-se a Ata de Registro de Preços para efetuar as contratações. Esse procedimento dá agilidade ao andamento do processo e permite que se atenda o Judiciário, diminuindo-se o número de aquisições emergenciais. Além da economia de escala, não há necessidade de 
manutenção de estoques elevados, evitando-se os custos decorrentes do armazenamento e o risco de perdas ou desperdício.

Nos casos de inexigibilidade de licitação, não cabe à administração decidir pela abertura de licitação, porque é inviável a competição. O Artigo 25 da Lei 8666/93 dispõe os casos exemplificativos dessa situação de inviabilidade de competição:

para aquisição de materiais, equipamentos, ou gêneros que só possam ser fornecidos por produtor, empresas ou representante comercial exclusivo, vedada a preferência de marca, devendo a comprovação de exclusividade ser feita através de atestado fornecido pelo órgão de registro do comércio do local em que se realizaria a licitação ou a obra ou o serviço, pelo Sindicato, Federação ou Confederação patronal, ou, ainda, pelas entidades equivalentes.

Portanto, se demonstrada a inviabilidade de competição, em especial nos casos de produto de fabricação exclusiva, sendo comprovada a exclusividade de fabricação do princípio ativo, considerando que é vedada preferência de marca, existe fundamento legal para a aquisição (Brasil, 1993; CONASS, 2007).

O fato de a aquisição ser feita para atender a uma determinação judicial, não exime a administração de cumprir as determinações da Lei de Licitações e Contratos Administrativos. Essa lei fixa os requisitos mínimos para contemplar tais situações, os quais devem ser cumpridos: a caracterização da situação emergencial é feita pela determinação judicial; a razão da escolha do fornecedor deve constar do processo, quer seja ele o que ofereceu o menor preço e prazo de entrega em uma consulta a todos os fabricantes que possuem registro e comercializem o produto, quer seja ele exclusivo, mediante a apresentação dos documentos que comprovem que apenas essa empresa pode fornecer o medicamento. Essa documentação deve ser anexada ao processo (CONASS, 2007).

Instruído o processo de compra, ainda é necessária a reserva orçamentária para cobrir a despesa. Após isso, é formalizado o ato de dispensa ou de inexigibilidade da licitação, encaminhando-se o processo à autoridade superior àquela que elaborou esse ato para ratificação da decisão e posterior publicação na Imprensa Oficial, nos prazos previstos no Artigo 26 da Lei 8666/93. Publicado o ato de ratificação, emite-se a nota de empenho e o pedido de compras (Brasil, 1993; CONASS, 2007).

Os gestores do SUS, em suas compras públicas de medicamentos por licitações e por mandados judiciais, estão obrigados a cumprir a legislação reguladora 
do mercado farmacêutico da Câmara de Regulação do Mercado de Medicamentos (CMED), tendo em vista que o valor de comercialização dos medicamentos registrados no Brasil obedece a regulamentação estabelecida por meio da Lei n10.742, de 6 de outubro de 2003, Decreto no 4.766, de 26 de junho de 2003, Resolução nํ 4, de 9 de março de 2011, Resolução n 3, de 2 de março de 2011, entre outras. Devem verificar se os preços ofertados no certame estão dentro do teto de preço pelo qual um laboratório ou distribuidor de medicamentos pode comercializar no mercado brasileiro um medicamento, tanto ao setor público como ao setor privado. Para tanto os gestores de saúde devem consultar os preços que são divulgados pela Secretaria-Executiva da CMED disponibilizados no sítio eletrônico da Anvisa. A divulgação é feita através de uma lista com os preços de todos os medicamentos que estão em conformidade com a legislação da CMED, atualizada mensalmente.

Os gestores de saúde devem ainda observar se o medicamento que está sendo adquirido consta do rol de produtos que são aplicados o Coeficiente de Adequação de Preços (CAP). Este é um desconto mínimo obrigatório, definido em um percentual (atualmente de $24,38 \%$ ) e incidente sobre o Preço Fábrica (PF) nas compras realizadas pelos entes da Administração Pública Direta e Indireta da União, dos Estados, do Distrito Federal e dos Municípios, quando adquiridos junto à empresa produtora, distribuidoras, farmácias e drogarias. A aplicação do CAP resultará no Preço Máximo de Venda ao Governo (PMVG).

O CAP é aplicado de duas maneiras distintas. Uma imediata, para as compras públicas motivadas por demandas judiciais, tendo em vista a natureza emergencial da compra, resultante de uma decisão judicial, e a outra para um rol de produtos em cujos preços serão submetidos ao CAP. Este rol é aprovado pelo Comitê TécnicoExecutivo da CMED, que têm a competência para inclusão ou não dos produtos na relação de que trata o art. $2^{\circ}$ da Resolução CMED nํㅜ 3, de 2 de março de 2011.

Os gestores de saúde devem ainda observar que alguns medicamentos são isentos de ICMS, ou seja, são contemplados por algum convênio do Conselho Nacional de Política Fazendária (CONFAZ), neste caso o Preço Fábrica e o PMVG devem ser calculados aplicando-se a desoneração do imposto.

Quando uma empresa produtora de medicamentos, distribuidora, farmácia ou drogaria ofertar para um medicamento preço superior ao permitido pela CMED, 
deverá ser encaminhada denúncia, acompanhada de todos os documentos comprobatórios, à Secretaria da CMED, que é o órgão competente para instaurar processo administrativo no caso de infração à legislação e ao Ministério Público Federal e Estadual conforme determina o Acórdão no 1437/2007 - Plenário, do Tribunal de Contas da União:

9.2. determinar ao Ministério da Saúde que dê ampla divulgação junto aos órgãos e entidades federais que fazem aquisições de medicamentos para atendimento da população, bem como junto às secretarias estaduais e municipais de saúde, acerca do teor das Resoluções da Câmara de Regulação do Mercado de Medicamentos - CMED nos 2/2001 e 4/2006, bem como da Orientação Interpretativa no 02/2006, da mesma Câmara, com vistas a alertar os gestores estaduais e municipais que, em caso de não observância das resoluções pelos fornecedores de medicamentos quando de compras efetuadas pelo setor público, deverá o gestor comunicar o fato à CMED e ao Ministério Público Federal e Estadual, sob pena de responsabilização por aquisição antieconômica e pela devolução dos recursos pagos acima do teto estabelecido pelos normativos da CMED, mediante instauração de tomada de contas especial.

\section{O papel do administrador público nas demandas judiciais da assistência farmacêutica}

Certamente se uma das causas que dão origem ao fornecimento de medicamento por demanda judicial é a indisponibilidade do medicamento padronizado para fornecimento ao usuário, a adoção de estratégias para manutenção de estoques regulares é fundamental. Essas estratégias passam pela adequada seleção do elenco de medicamentos padronizados no SUS, uma programação com base em dados confiáveis, agilidade na aquisição e fiscalização na execução dos contratos de fornecimento (CONASS, 2007).

Ressalta-se a importância de se realizar uma previsão orçamentária adequada à realidade e disponibilizar os recursos financeiros específicos para atendimento dessas demandas. Se forem utilizados os recursos destinados às aquisições rotineiras de medicamentos para os casos de ações judiciais, fica estabelecido um ciclo no qual a indisponibilidade de recursos para efetivar essas compras, leva à falta de medicamentos que estão sob responsabilidade da Secretaria de Saúde, com consequente aumento de ações judiciais que assegurem o acesso dos usuários ao medicamento (CONASS, 2007). 
O acompanhamento dos projetos de lei locais, bem como a atualização das informações relativas a novas normas federais, é fundamental para que a administração tenha uma ação pró-ativa, no sentido de incorporar novas demandas no seu planejamento, evitando futuras ações judiciais (Brasil, 2006; CONASS, 2007).

Outras medidas que devem ser adotadas pelo gestor e, recomendadas pelo Ministério Público é a promoção do uso racional de medicamentos, bem como a orientação dos profissionais médicos, no âmbito do SUS, a observarem os Protocolos Clínicos e Diretrizes Terapêuticas e a prescreverem adotando, obrigatoriamente, a Denominação Comum Brasileira (DCB) ou, na sua falta, da Denominação Comum Internacional (DCl).

A realização de seminários, palestras e debates sobre o tema, com as instituições envolvidas no processo, desde as Procuradorias Gerais dos Estados e Municípios, Ministérios Públicos Estadual e Federal e o Poder Judiciário tem contribuído na solução ou minimização das ações judiciais. Porém, também são necessárias ações que envolvam: atualização permanente dos protocolos; avaliação de novas tecnologias; análise da relação custo/benefício, além de outros aspectos relacionados a farmacoeconomia; promoção do uso racional de medicamentos, com indicação, prescrição e dispensação respaldados em critérios técnicos; padronização e fornecimento de medicamentos respaldados em Protocolos Clínicos e Diretrizes Terapêuticas; inclusões de medicamentos com registro na Anvisa; constituição de Comissão de Especialistas para se manifestarem durante a tramitação dos processos judiciais que demandem o fornecimento de medicamentos do componente especializado; e divulgação das decisões judiciais para os gestores (CONASS, 2007).

Não há dúvidas que o fortalecimento da Assistência Farmacêutica no SUS é um dos principais caminhos para a minimização da judicialização da saúde e ao acesso aos tratamentos pelo SUS. Esse fortalecimento envolve, sobretudo, a qualificação da gestão e dos serviços, a disponibilização de um rol abrangente de medicamentos padronizados, o qual está atrelado ao financiamento adequado, entre outros pontos. 


\section{Considerações finais}

Como o direito à saúde deve ser garantido mediante políticas públicas, faz-se necessário para tanto, à luz da melhor e mais forte evidência disponível de eficácia, segurança, efetividade, custo-efetividade e, desde que a sociedade possa arcar com os custos, selecionar os recursos terapêuticos que possibilitem cobertura qualitativa completa para o tratamento das doenças. Essa é condição fundamental para que o poder público sustente o argumento de que esse direito só pode mesmo ser garantido mediante políticas públicas e possa fazer frente à grande quantidade de ações judiciais que não discriminam a garantia de direitos (demandas por recursos terapêuticos que constam nas políticas ou para doenças não cobertas por política) e a demanda/consumo de tecnologias específicas, ainda que o tratamento da doença esteja previsto em política terapêutica com o uso de outras tecnologias (Vieira, Zucchi, 2009).

Não se pode ignorar, no entanto, que, a interpretação dos direitos sociais apenas na dimensão individual, desconsiderando a dimensão coletiva, não permite considerar o problema em todas as suas dimensões e em toda a sua complexidade, o que resulta em medidas que em vez de promoverem a justiça social acabam prolongando a dívida social com a parcela mais vulnerável da população (Chieffi, Barata, 2009).

Pode-se inferir, contudo, que a via judicial tem propiciado o exercício dos princípios constitucionais da universalidade, integralidade e equidade na assistência à saúde. Porém, quando se analisa de forma mais ampla, percebe-se uma lógica adversa: se, por um lado, as decisões judiciais visam a garantir o atendimento ao direito e às necessidades do cidadão, por outro, remetem ao gestor a responsabilidade de decisões administrativas que muitas vezes contradizem os princípios constitucionais da equidade e acesso à assistência à saúde de qualidade (Souza et al., 2011).

Constitui dever do Estado fornecer todos os medicamentos disponíveis no mercado mundial? É legítimo ao Estado adotar uma política de fornecimento de medicamentos que extrapole suas possibilidades orçamentárias? Se legítimo e possível, cabe ao Poder Judiciário conceder tais medicamentos a alguns poucos demandantes ou o Estado deve promover um sistema de saúde, baseado em 
políticas públicas de forma a atender a demanda social por esse direito? (Mattos; Souza, 2011).

Sabe-se que a prestação de assistência à coletividade se dá por meio das políticas públicas, que objetivam atender as principais necessidades da população de forma equânime, tendo como base para definição de suas ações o direito à saúde, direito este consagrado pela Constituição Federal de 1988. Apesar do anseio de que o Brasil possa oferecer tudo o que existe de mais moderno e necessário à garantia da vida e da saúde, é forçoso reconhecer que incumbe ao Poder Legislativo e ao Poder Executivo traçar as políticas públicas de saúde, nas quais, necessariamente, está incluída a opção de selecionar os medicamentos que serão oferecidos universalmente a toda população, e assim, apenas nos casos de omissão ou na insuficiência de tais políticas o Poder Judiciário estaria autorizado a intervir (Mattos; Souza, 2011; Araújo et al., 2013).

Como desdobramento de audiência pública realizada pelo Supremo Tribunal Federal em 2009, o Conselho Nacional de Justiça recomendou aos tribunais a constituição de apoio técnico (médico e farmacêutico) aos magistrados. Isso auxiliaria na formação de juízo de valor quanto à apreciação das questões clínicas para a tomada de decisão do fornecimento de serviços de saúde pela via judicial. Esta abordagem pode auxiliar na busca do equacionamento dos dilemas enfrentados, sobretudo em relação ao confronto entre o coletivo versus o individual, visando uma forma mais justa de resolução dos problemas em relação à dificuldade de acesso a assistência terapêutica (CNJ, 2010; Macedo, Lopes, Barberato-Filho, 2011; Souza et al., 2011).

A construção normativa de uma sociedade pressupõe o conflito de interesses e, do mesmo modo, a demanda judicial reflete disputas, conquistas ou retrocessos, estabelecidos para além da esfera jurídica. O grande desafio é pensar na judicialização da saúde como estratégia legítima, porém a ser orquestrada com outros mecanismos de garantia constitucional de saúde para todos. As demandas judiciais não podem ser consideradas como principal instrumento deliberativo, pois, de fato, para o alcance da justiça, deve ser adotado um conjunto de ações por meio das quais se busque implementar as diretrizes constitucionais (Ventura et al, 2010).

Em sociedades em desenvolvimento como a brasileira, a garantia dos direitos coletivos em saúde deve prevalecer sobre os direitos individuais. A distância entre 
direitos coletivos e individuais tende a se reduzir na medida em que aumentam os recursos disponíveis para o financiamento da saúde, permitindo atender a um maior gradiente de necessidades de saúde coletivas, mesmo que estas se expressem de forma pouco frequente em termos individuais. Portanto, a garantia de recursos estáveis para o financiamento da saúde e a redução das brechas de equidade, seja por meio da ampliação das fontes de financiamento, seja pela melhoria da eficiência no uso dos recursos, serão sempre determinantes de parte das conquistas futuras que permitirão a aproximação entre as perspectivas individuais e coletivas em matéria de direito sanitário (Medici, 2010).

\section{Referências}

ALBUQUERQUE, R. B.; CORDEIRO, M.; LINS, A. C. V.; NEIVA, J. S. M.; ROCHA, M. S. Parecer n. 1300/2006-AGU/CONJUR-MS/JSMN. Parecer de consolidação da Assistência Farmacêutica, 2006. In: CONASS, Conselho Nacional de Secretários de Saúde. Assistência Farmacêutica no SUS. Brasília: CONASS, 2007. 186 p.

ANDRADE, E. G.; MACHADO, C. D.; FALEIROS, D. R.; SZUSTER, D. C,; GUERRA JÚNIOR, A. A.; SILVA, G.D.; CHERCHIGLIA, M. L.; ACÚRCIO, F. A. A judicialização da saúde e a política nacional de assistência farmacêutica no Brasil: gestão da clínica e medicalização da justiça. Rev. Med. Minas Gerais, 18(4):S46-S50, 2008.

ARAÚJO, L.M.; FRAGA, A. J. A.; AGUIAR NETA, A. M., DE SOUZA, L.R.B. Judicialização da saúde: uma revisão da literatura. Rev. Enferm. UFPI. 2(2): 49-54, 2013.

BORGES, D. C. L.; UGA, M. A. D. Conflitos e impasses da judicialização na obtenção de medicamentos: as decisões de $1^{\underline{a}}$ instância nas ações individuais contra o Estado do Rio de Janeiro, Brasil, em 2005. Cad. Saúde Pública, 26(1):59-69, 2010.

BRASIL, Ministério da Saúde. Aquisição de Medicamentos para a Assistência Farmacêutica no SUS. Brasília: Ministério da Saúde, 2006a. 47p.

BRASIL, Ministério da Saúde. Assistência Farmacêutica na atenção básica. Instruções técnicas para sua organização. Brasília: Ministério da Saúde, 2006b. 98p.

BRASIL, Ministério da Saúde. Orientação aos gestores federal, estadual e municipal do Sistema Único de Saúde. Brasília: Ministério da Saúde, 2012. [on line] Disponível em: $\quad$ http://portal.saude.gov.br/portal/arquivos/pdf/Orientacao Acordao 1146.pdf. Acesso em 31 de agosto de 2013.

BRASIL. Constituição (1988). Constituição da República Federativa do Brasil. Brasília, DF: Senado Federal, 1988.

BRASIL. Lei n. 8.666, de 21 de junho de 1993. Regulamenta o art. 37, inciso XXI, da Constituição Federal, institui normas para licitações e contratos da Administração Pública e dá outras providências. 1993. Disponível em: http://www.planalto.gov.br/ccivil_03/Leis/L8666cons.htm. Acesso em: 8 nov. 2013. 
CHIEFFI, A.L.I; BARATA, R.B. Ações judiciais: estratégia da indústria farmacêutica para introdução de novos medicamentos. Revista de Saúde Pública. 44(3): 421-429, 2010.

CHIEFFI, A.L.I; BARATA, R.B. Judicialização da política pública de assistência farmacêutica e equidade. Cadernos de Saúde Pública, 25(8):1839-1849, 2009.

CNJ - Conselho Nacional de Justiça. Recomendação n 1, de 30 de março de 2010. Recomenda aos Tribunais a adoção de medidas visando a melhor subsidiar os magistrados e demais operadores do direito, para assegurar maior eficiência na solução das demandas judiciais envolvendo a assistência à saúde. Diário da Justiça. 07 abr 2010:4-6.

CONASS, Conselho Nacional de Secretários de Saúde. Assistência Farmacêutica no SUS. Brasília: CONASS, 2007. 186 p.

http://www.cnj.jus.br/images/stories/docs_cnj/recomendacoes/reccnj_31.pdf

MACEDO, E.I; LOPES, L.C.; BARBERATO-FILHO, S. Análise técnica para a tomada de decisão do fornecimento de medicamentos pela via judicial. Revista de Saúde Pública, São Paulo; 45(4):706-713, 2011.

MARIN, N.; LUIZA, V.L.; OSORIO-DE-CASTRO, C.G.S.; MACHADO-DOS-SANTOS, S. Assistência farmacêutica para gerentes municipais de saúde. Rio de Janeiro: OPAS/OMS, 2003. 373p.

MATTOS, K.D.G.; SOUZA, G.A. Ativismo Judicial e Direito à Saúde: uma Análise da Tutela Jurisdicional nas Ações de Medicamentos. Direito Público, 1(37):7-23, 2011.

MEDICI, A. C. Judicialização, integralidade e financiamento da saúde. Diagn Tratamento; 15(2):81-7, 2010.

OPAS - Organização Pan-Americana da Saúde. Atenção Farmacêutica no Brasil: "Trilhando Caminhos". Brasília: Organização Pan-Americana da Saúde, 2002. 24p.

SOUZA, I. F.; BRZOZOWSKI, F. S.; SAVI, L. A.; BASTOS, P. R. H. O. Acesso à assistência terapêutica no SUS: Implicações da bioética na judicialização da saúde. In: OLIVEIRA BASTOS, Paulo Roberto Haidamus de et al. (Org.). Reflexões Interdisciplinares: A Ética em Evidência. Curitiba: Editora CRV, 2011, v. 1, p. 43-61.

VIEIRA, F.S; ZUCCHI, P. Demandas judiciais e assistência terapêutica no Sistema Único de Saúde. Revista da Associação Médica Brasileira; 55(6):672-683, 2009. 